\title{
DEVELOPMENTAL DELAY AND IN UTERO PHENYTOIN EXPOSURE
}

Psychomotor development was assessed in preschool children exposed to antiepileptic drugs (AED) in utero as part of a population-based longitudinal follow-up study of children born to women with meticulously treated epilepsy during pregnancy at the Karolinska Institute, Stockholm, Sweden. Exposed $(n=67)$ and unexposed $(n=66)$ children were tested with the Griffiths' test at 4.5-5 yrs of age in 6 subsets: locomotor function, personal and social behavior, hearing and speech, eye and hand coordination, performance, and practical reasoning. The Griffiths' test evaluates the child's behavior and is widely used in Sweden as the standardized measure of psychomotor development in preschool children. Global scores showed no significant differences in the two groups, but exposed children obtained slightly lower scores in 4 of the 6 subtests. Children exposed to phenytoin in utero $(n=16)$ showed a significant but subtle reduction in locomotor function. Carbamazepine in utero $(n=35)$ had no measurable adverse effect. The majority $(85 \%)$ received monotherapy in low doses. Exposed children had significantly fewer siblings $(\mathrm{p}<0.01)$, and AED-treated mothers were more likely to have lower levels of education $(p<0.001)$ than the unexposed group. Socioeconomic status was otherwise similar in the two groups. The first evaluation of this cohort of children had shown a significant increase in minor anomalies in the exposed group, but psychomotor development at 9 mos of age had revealed no differences in the scores of the 2 groups. (Wide K, Henning E, Tomson T, Winbladh B. Psychomotor development in preschool children exposed to antiepileptic drugs in utero. Acta Pediatr 2002;91:409-414). (Respond: Dr K Wide, Department of Clinical Sciences, Division of Pediatrics, Karolinska Institutet, B57, Huddinge University Hospital, SE-141 46 Stockholm, Sweden).

COMMENT. Psychomotor development, especially locomotor function, shows significant but subtle delays in preschool children exposed in utero to phenytoin but not carbamazepine. Previous reports, cited by the authors, have found evidence of developmental delay in children exposed to carbamazepine. The differences between AED-exposed and unexposed children are not observed when tested at 9 months of age, but could be more obvious at school age. Further followup of the cohort is planned.

\section{TEST FOR VIGABATRIN-INDUCED FIELD DEFECTS IN CHILDREN}

A visual-evoked potential (VEP) technique for identifying visual field defects in children with epilepsy treated with vigabatrin has been developed at Aston University, Birmingham, and Cardiff University, UK. The VEP was field specifric with a central (0-5 degree radius) and peripheral stimulus (30-60 degree radius). Black and white checks used as stimuli increased in size with eccentricity and reversed at different rates, allowing a record of separate central and peripheral responses. Initially, five vigabatrin-treated young adults with field defects were identified using this technique and were examined with electroretinograms (ERG). Of 39 children aged 3 to 15 years treated with vigabatrin, 35 complied with the field-specific stimulus, 26 complied with ERG, and 12 with perimetry. The field-specific stimulus identified 3 of 4 abnormal and 7 of 8 normal perimetry results, with good sensitivity (75\%) and specificity ( $87.5 \%)$. A typical vigabatrin visual field loss occurred in $25.7 \%$ of children treated, similar to the prevalence in adults. Combined with the ERG 30- Hz flicker amplitude, the field specific stimulus is a satifactory method for identifying VGB-induced visual field loss in children older than 2 and under 10 years of age. (Harding GFA, Spencer EL, Wild JM et al. Field-specific visual-evoked potentials. Identifying field defects in vigabatrin-treated children. Neurology April (2 of 2) 2002;58:1261- 